PROCEEDINGS OF THE

AMERICAN MATHEMATICAL SOCIETY

Volume 137, Number 7, July 2009, Pages 2293-2298

S 0002-9939(09)09703-2

Article electronically published on February 20, 2009

\title{
PERTURBATIVE SOLUTIONS TO THE EXTENDED CONSTANT SCALAR CURVATURE EQUATIONS ON ASYMPTOTICALLY HYPERBOLIC MANIFOLDS
}

\author{
ERWANN DELAY
}

(Communicated by Matthew J. Gursky)

\begin{abstract}
The extended constant scalar curvature equations is a particular case of the conformal contraint equations introduced by H. Friedrich. It was first studied by A. Butscher in an asymptotically flat setting. We prove the local existence of solutions to the extended constant scalar curvature equations near some asymptotically hyperbolic Einstein metrics. This gives a new local construction of asymptotically hyperbolic metrics with constant scalar curvature.
\end{abstract}

\section{INTRODUCTION}

The study of constant scalar curvature metrics plays a particulary important part in Riemannian geometry and in general relativity. For instance in the Riemannian point of view it gives rise to the well-known Yamabe problem when working in a conformal class. For general relativity, constant scalar curvature Riemannian metrics are particular solutions to the constraint equations, for instance in the timesymmetric case, the constraint equations being constraints on the initial data for Einstein equations (see [6 for instance). In [4, 5], A. Butscher studied a system of equations called the "extended constraint equations", which are equivalent to the usual constraints. This new system has the advantage that, as in the case of the Einstein equation for Riemannian metrics, it can naturally be modified to a determined elliptic system by adding a gauge-breaking term.

In the asymptotically flat setting, A. Butscher proves in [4, [5] that this system can be solved near the usual Euclidean metric.

The present note is a study of the extended constraint equations on asymptotically hyperbolic (A.H.) manifolds, in the time-symmetric case, near an Einstein metric. The problem we are studying here is then reduced to a purely Riemannian problem of scalar curvature. It will appear that the proof is more simple in the A.H. context due to the fact we do not have to handle a cokernel. Let us now introduce some notation and the system we want to study. For a Riemannian metric $g$ on a manifold $M^{n}$, let us denote by $R(g)$ the scalar curvature of $g$. In the asymptotically

Received by the editors March 17, 2008.

2000 Mathematics Subject Classification. Primary 35J50, 58J05, 35J70, 35J60, 35Q75.

Key words and phrases. Asymptotically hyperbolic manifolds, general relativity, constraint equations, symmetric 2-tensor, asymptotic behavior. 
hyperbolic setting, the constant scalar curvature equation is

$$
R(g)=-n(n-1) .
$$

The extended scalar curvature equation is

$$
\begin{aligned}
& \operatorname{Ric}(g)+(n-1) g=S, \\
& \operatorname{div}_{g} S=0,
\end{aligned}
$$

where, for a metric $g$, a symmetric two-tensor $T$ and a one-form $\xi$,

$$
S=T-\frac{1}{n} \operatorname{Tr}_{g}(T) g+\dot{\mathcal{L}} \xi
$$

is a trace-free symmetric two-tensor and $\stackrel{\circ}{\mathcal{L}}$ is the conformal killing operator (see section 21). At this stage it is important to remark that equation (1.3) is a consequence of (1.2) by the Bianchi identity. Taking the trace of equation (1.2) we see that any solution $g$ has constant scalar curvature satisfying (1.1). Reciprocally any metric of constant scalar curvature $R(g)=-n(n-1)$ is a solution of (1.2) with, for instance, $\xi=0$ and $T=\operatorname{Ric}(g)+(n-1) g 1$

We fix a smooth asymptotically hyperbolic metric $g_{0}$. The weighted Hölder spaces $C_{s}^{k, \alpha}$ we will work with are the ones used by Lee [9] $2 C_{s}^{k, \alpha}$ consist of tensor fields of the form $v=\rho^{s} u$ for $u$ in the usual Hölder space $C^{k, \alpha}$, with the norm

$$
\|v\|_{C_{s}^{k, \alpha}}=\left\|\rho^{-s} v\right\|_{C^{k, \alpha}},
$$

$\|.\|_{C^{k, \alpha}}$ being the usual Hölder norm relative to the metric $g_{0}$.

The theorem we will prove is the following.

Theorem 1.1. Let $\left(M^{n}, g_{0}\right)$ be a non-degenerate asymptotically hyperbolic Einstein manifold of dimension $n \geq 2$. Let $s \in(0, n-1), k \in \mathbb{N} \backslash\{0\}$ and $\alpha \in(0,1)$. For all sufficiently small $T \in C_{s}^{k, \alpha}$, there exists $(\xi, h)$ close to zero in $C_{s}^{k+1, \alpha} \times C_{s}^{k+2, \alpha}$ such that $g=g_{0}+h$ and $\xi$ solves (1.2) and (1.3) (then $g$ solves (1.1)). The map

$$
\begin{aligned}
\mathcal{C}: C_{s}^{k, \alpha} & \longrightarrow C_{s}^{k+1, \alpha} \times C_{s}^{k+2, \alpha} \\
T & \mapsto(\xi, h)
\end{aligned}
$$

is smooth near zero.

Here we say that the metric $g$ is non-degenerate if the $L^{2}$-kernel of $\Delta_{L}+2(n-1)$ acting on a trace-free symmetric two-tensor field is trivial, $\Delta_{L}$ being the Lichnerowicz Laplacian of $g$ (see section 21). This condition is satisfied for instance on the hyperbolic space but also on a large class of A.H. manifolds (see [9] and Theorem 2.2 of [1]).

With the construction we use, the metric $g$ we obtain also satisfies the harmonic gauge; that is, the identity map from $(M, g)$ to $\left(M, g_{0}\right)$ is harmonic. The construction also proves that the solution $g$ of (1.2) and (1.3) is locally unique (near $g_{0}$ ) in the harmonic gauge. Finally we mention that initial data in the spatial harmonic gauge, as the ones constructed here, are of interest to some stability results 3 .

\footnotetext{
${ }^{1}$ Of course, we can also take $T=\operatorname{Ric}(g)$, but our choice here is more natural in the A.H. context.

${ }^{2}$ As the reference [9] will be used several times in the paper, it is important to remark that the dimension $n+1$ there corresponds to $n$ here.
} 


\section{Definitions, notation and Conventions}

Let $(\bar{M}, g)$ be a smooth, compact $n$-dimensional manifold with boundary $\partial_{\infty} M$. Let $M:=\bar{M} \backslash \partial_{\infty} M$, which is a non-compact manifold. We call $\partial_{\infty} M$ the boundary at infinity of $M$. Let $g$ be a Riemannian metric on $M$. We say that $(M, g)$ is conformally compact if there exists a smooth defining function $\rho$ on $\bar{M}$ (that is, $\rho \in C^{\infty}(\bar{M}), \rho>0$ on $M, \rho=0$ on $\partial_{\infty} M$ and $d \rho$ is nowhere vanishing on $\left.\partial_{\infty} M\right)$ such that $\bar{g}=\rho^{2} g$ is a smooth Riemannian metric on $\bar{M}$. If $|d \rho|_{\bar{g}}=1$ on $\partial_{\infty} M$, it is well known that $g$ has asymptotically sectional curvature -1 (see [10] for example) near its boundary at infinity. In this case we say that $(M, g)$ is asymptotically hyperbolic.

If we assume moreover that $g$ has constant scalar curvature, then asymptotic hyperbolicity enforces the normalisation

$$
R(g)=-n(n-1),
$$

where $R(g)$ is the scalar curvature of $g$. Also, if $g$ is Einstein, then the Ricci curvature of $g$ is

$$
\operatorname{Ric}(g)=-(n-1) g .
$$

We denote by $\nabla$ the Levi-Civita connection of $g$ and by Riem $(g)$ the Riemannian sectional curvature of $g$.

We denote by $\mathcal{T}_{p}$ the set of rank $p$ covariant tensors. When $p=2$, we denote by $\mathcal{S}_{2}$ the subspace of symmetric tensors which splits as $\mathcal{S}_{2}=\mathcal{G} \oplus \dot{\mathcal{S}}_{2}$, where $\mathcal{G}$ is the set of scalar multiples of $g$ and $\dot{\mathcal{S}}_{2}$ is the set of trace-free tensors (relative to $g$ ). We observe the summation convention (the corresponding indices run from 1 to $n$ ), and we use $g_{i j}$ and its inverse $g^{i j}$ to lower or raise indices. For instance, we have

$$
\operatorname{Ric}(g)_{i j}=g^{k l} \operatorname{Riem}(g)_{k i l j} .
$$

The Laplacian is defined as

$$
\triangle_{g}=-\operatorname{Tr}_{g} \nabla^{2}=\nabla^{*} \nabla
$$

where $\nabla^{*}$ is the $L^{2}$ formal adjoint of $\nabla$. The Lichnerowicz Laplacian acting on a symmetric covariant two-tensor field is

$$
\triangle_{L}=\triangle_{g}+2(\text { Ric }- \text { Riem })
$$

where

$$
(\operatorname{Ric} u)_{i j}=\frac{1}{2}\left[\operatorname{Ric}(g)_{i k} u_{j}^{k}+\operatorname{Ric}(g)_{j k} u_{i}^{k}\right]
$$

and

$$
(\operatorname{Riem} u)_{i j}=\operatorname{Riem}(g)_{i k j l} u^{k l} .
$$

For $u$ a covariant two-tensor field on $M$ we define the divergence of $u$ by

$$
\left(\operatorname{div}_{g} u\right)_{i}=-\nabla^{j} u_{j i}
$$

For a one-form $\omega$ on $M$, we define the divergence of $\omega$ :

$$
d^{*} \omega=-\nabla^{i} \omega_{i},
$$

the symmetric part of its covariant derivative:

$$
\left(\mathcal{L}_{g} \omega\right)_{i j}=\frac{1}{2}\left(\nabla_{i} \omega_{j}+\nabla_{j} \omega_{i}\right)
$$


(note that $\mathcal{L}_{g}^{*}=\operatorname{div}_{g}$ ), and the trace-free part of that last tensor:

$$
\left(\stackrel{\circ}{\mathcal{L}}_{g} \omega\right)_{i j}=\frac{1}{2}\left(\nabla_{i} \omega_{j}+\nabla_{j} \omega_{i}\right)+\frac{1}{n}\left(d^{*} \omega\right) g_{i j} .
$$

The operator $\mathcal{L}$ is sometimes called the conformal killing operator. Its formal $L^{2}$ adjoint acting on trace-free symmetric two-tensors is $\dot{\mathcal{L}}_{g}^{*}=\operatorname{div}_{g}$.

\section{Gauge-broken equation And Proof}

It is well known that the system (1.2)-(1.3) is not elliptic because of the invariance by diffeomorphism. As usual in this context, we will add a gauge term to the system in such a way that it becomes elliptic and such that the solutions to the new system are solutions to the original one.

Let us define an operator from symmetric two-tensors to one-forms :

$$
B_{g}(h)=\operatorname{div}_{g}\left(h-\frac{1}{2}\left(\operatorname{Tr}_{g} h\right) g\right)=\operatorname{div}_{g} h+\frac{1}{2} d\left(\operatorname{Tr}_{g} h\right) .
$$

The new system we consider is

$$
\begin{aligned}
& \operatorname{Ric}(g)+(n-1) g-\mathcal{L}_{g}\left(B_{g}\left(g_{0}\right)\right)=S, \\
& \operatorname{div}_{g} S=0,
\end{aligned}
$$

where $S$ is as in (1.4). First let us verify that the solutions to the new system are solutions to the original one.

Proposition 3.1. Let $s \in[0, n), k \in \mathbb{N} \backslash\{0\}, \alpha \in(0,1)$. Let $g_{0}$ be an A.H. metric with negative Ricci curvature. If $h \in C_{s}^{k+2, \alpha}$ is sufficiently small, and the metric $g=g_{0}+h$ is a solution of (3.1)-(3.2), then it is a solution of (1.2) -(1.3) .

Proof. We will apply the operator $B_{g}$ to the equation (3.1). We remark that $\operatorname{div}_{g} S=\operatorname{Tr}_{g} S=0$, that $B_{g}(\operatorname{Ric}(g))=0$ by the Bianchi identity, and that $B_{g}(g)=0$. Then, if we denote by $\omega$ the one-form $B_{g}\left(g_{0}\right)$, we obtain that

$$
B_{g}\left(\mathcal{L}_{g} \omega\right)=0 .
$$

This equation reads in local coordinates:

$$
-\nabla^{i}\left[\frac{1}{2}\left(\nabla_{i} \omega_{j}+\nabla_{j} \omega_{i}\right)\right]+\frac{1}{2} \nabla_{j} \nabla^{i} \omega_{i}=0 .
$$

Commuting derivatives and multiplying by 2 , we obtain that

$$
\Delta_{g} \omega-\operatorname{Ric}(g) \omega=0 .
$$

As $\operatorname{Ric}\left(g_{0}\right)$ is negative, the operator $\Delta_{g_{0}}-\operatorname{Ric}\left(g_{0}\right)$ has no $L^{2}$ kernel on one-forms, so from [9, Theorem C, Lemmas 7.2 and 7.10, and Corollary 7.4], it is an isomorphism from $C_{t}^{k+1, \alpha}$ to $C_{t}^{k-1, \alpha}$, for all $t \in(-1, n)$ (the reader can see the proof of proposition A.2 in [1] for more details). Now if $h$ is small in $C_{s}^{k+2, \alpha}$, then it is small in $C_{0}^{k+2, \alpha}$, so the operator $\Delta_{g}-\operatorname{Ric}(g)$ is still an isomorphism between the same spaces. As $\omega \in C_{s}^{k+1, \alpha}$ with $s \in[0, n)$, we conclude that $\omega=0$.

Remark. The fact that $B_{g}\left(g_{0}\right)$ vanishes shows that the identity map from $(M, g)$ to $\left(M, g_{0}\right)$ is harmonic (see [8], for instance).

Let us now construct solutions to the system (3.1)-(3.2) by an implicit function theorem on Banach spaces. 
Proposition 3.2. Let $s \in(0, n-1), k \in \mathbb{N} \backslash\{0\}, \alpha \in(0,1)$. Let $g_{0}$ be a nondegenerate A.H. Einstein metric. Then for all $T \in C_{s}^{k, \alpha}$ sufficiently small, there exists a unique $(\xi, h)$ close to zero in $C_{s}^{k+1, \alpha} \times C_{s}^{k+2, \alpha}$ such that $g=g_{0}+h$ and $\xi$ solve (3.1) -(3.2).

Proof. Let us consider the map from a neighborhood of zero in $C_{s}^{k+2, \alpha} \times C_{s}^{k+1, \alpha} \times$ $C_{s}^{k, \alpha}$ to $C_{s}^{k, \alpha} \times C_{s}^{k-1, \alpha}$ defined by

$$
F(h, \xi, T):=\left(\begin{array}{c}
\operatorname{Ric}(g)+(n-1) g-\mathcal{L}_{g}\left(B_{g}\left(g_{0}\right)\right)-S \\
\operatorname{div}_{g} S
\end{array}\right),
$$

where $g=g_{0}+h$ and $S$ is defined by (1.4). The map $F$ is well defined and differentiable in a neighborhood of zero. The metric $g_{0}$ being Einstein, we have $F(0,0,0)=0$, and the derivative of $F$ in the first two variables at the origin is

$$
D_{(h, \xi)} F(0,0,0)(\delta h, \delta \xi)=\left(\begin{array}{c}
\frac{1}{2} \Delta_{L}(\delta h)+(n-1)(\delta h)-\dot{\mathcal{L}}(\delta \xi) \\
\operatorname{div} \circ \dot{\mathcal{L}}(\delta \xi)
\end{array}\right),
$$

where all the operators are relative to the metric $g_{0}$. The hypothesis that $g_{0}$ is non-degenerate together with the results of [9, Theorem $\mathrm{C}$ and Proposition D] give us that the operator $\frac{1}{2} \Delta_{L}+(n-1)$ is an isomorphism from $C_{s}^{k+2, \alpha}$ to $C_{s}^{k, \alpha}$ when $s \in(0, n-1)$. Now the operator $\mathcal{L}$ has no $L^{2}$ kernel ([2], [7]), so the same is true for the operator $\operatorname{div} \circ \dot{\mathcal{L}}$ because $\operatorname{div}=\dot{\mathcal{L}}^{*}$. Again the result of 9 , Theorem $\mathrm{C}$ and Proposition G] shows that the vector Laplacian div $\circ \mathcal{L}$ is an isomorphism from $C_{\sigma}^{k+1, \alpha}$ to $C_{\sigma}^{k-1, \alpha}$ when $\sigma \in(-1, n)$. We then conclude that $D_{(h, \xi)} F(0,0,0)$ is an isomorphism, and the proposition follows by the implicit function theorem.

Propositions 3.2 and 3.1 together prove the main theorem 1.1

\section{ACKNowledgments}

The author is grateful to A. Butscher, P. Chruściel, H. Friedrich, F. Gautero and the referee for their comments.

\section{REFERENCES}

1. M.T. Anderson, P.T. Chruściel, and E. Delay, Non-trivial, static, geodesically complete vacuum space-times with a negative cosmological constant. II: $n \geq 5$, Proceedings of the Strasbourg Meeting on AdS-CFT correspondence (Berlin, New York) (O. Biquard and V. Turaev, eds.), IRMA Lectures in Mathematics and Theoretical Physics, vol. 8, de Gruyter, 2005, pp. 165-204. MR 2160871 (2007b:53160)

2. L. Andersson and P.T. Chruściel, Solutions of the constraint equations in general relativity satisfying "hyperboloidal boundary conditions", Dissert. Math. 355 (1996), 100 pp. MR1405962 (97e:58217)

3. L. Andersson and V. Moncrief, Elliptic-hyperbolic systems and the Einstein equations, Annales Henri Poincaré 4 (2003), 1-34. MR1967177 (2004c:58060)

4. A. Butscher, Exploring the conformal constraint equations, The Conformal Structure of Space-Time, Lect. Notes in Phys., 604, Springer, Berlin, 2002, pp. 195-222. MR2007930 (2004j:83009)

5. - Perturbative solutions of the extended constraint equations in general relativity, Commun. Math. Phys. 272 (2007), no. 1, 1-23. MR2291799 (2008c:83006)

6. A. Chruściel and H. Friedrich (eds.), The Einstein equations and the large scale behavior of gravitational fields (50 years of the Cauchy problem in general relativity), Birkhäuser-Verlag, Basel-Boston-Berlin, 2004. MR2098911(2005f:83001)

7. R. Gicquaud, De l'équation de prescription de courbure scalaire aux équations de contrainte en relativité générale sur une variété asymptotiquement hyperbolique (2008), arXiv:0802.3279. 
8. C.R. Graham and J.M. Lee, Einstein metrics with prescribed conformal infinity on the ball, Adv. Math. 87 (1991), 186-225. MR1112625 (92i:53041)

9. J.M. Lee, Fredholm operators and Einstein metrics on conformally compact manifolds, Memoirs AMS 183 (2006), no. 864. MR2252687 (2007m:53047)

10. R. Mazzeo, The Hodge cohomology of a conformally compact metric, J. Diff. Geom. 28 (1988), 309-339. MR961517 (89i:58005)

Institut de Mathématiques et Modélisation de Montpellier, UMR 5149 CNRS, Université Montpellier II, Place Eugène Bataillon, 34095 Montpellier cedex 5, France Current address: Laboratoire d'Analyse Non linéaire et Géométrie (EA2151), Faculté des Sciences, 33 rue Louis Pasteur, F-84000 Avignon, France

E-mail address: Erwann.Delay@univ-avignon.fr

$U R L:$ http://www.math.univ-avignon.fr/Delay 